\title{
Preventive and Risk Reduction Strategies for Women at High Risk of Developing Breast Cancer: a Review
}

\author{
Arvind Krishnamurthy ${ }^{1 *}$, Viveka Soundara ${ }^{2}$, Vijayalakshmi Ramshankar ${ }^{3}$
}

\begin{abstract}
Breast cancer is the most commonly diagnosed invasive cancer among women. Many factors, both genetic and non-genetic, determine a woman's risk of developing breast cancer and several breast cancer risk prediction models have been proposed. It is vitally important to risk stratify patients as there are now effective preventive strategies available. All women need to be counseled regarding healthy lifestyle recommendations to decrease breast cancer risk. As such, management of these women requires healthcare professionals to be familiar with additional risk factors so that timely recommendations can be made on surveillance/risk-reducing strategies. Breast cancer risk reduction strategies can be better understood by encouraging the women at risk to participate in clinical trials to test new strategies for decreasing the risk. This article reviews the advances in the identification of women at high risk of developing breast cancer and also reviews the strategies available for breast cancer prevention.
\end{abstract}

Keywords: Breast cancer risk assessment - risk prediction - risk reduction - strategies

Asian Pac J Cancer Prev, 17 (3), 895-904

\section{Introduction}

Breast cancer is the most commonly diagnosed invasive cancer among women. Although once considered to be primarily a disease of Western women, $52 \%$ of new cases and $62 \%$ of deaths of breast cancer now occur in the developing countries. A recent review (De Santis et al., 2015) showed that the breast cancer incidence rates showed an increasing trend in most countries, including those with historically higher rates, such as Europe, as well as in many countries in Latin America, Asia, and Africa. (Regions which had historically lower incidence rates). The incidence rates were reportedly stable in North America and Oceania and have declined in a few other European countries and Israel.

The cause for the increase in breast cancer incidence is likely to be multifactorial. Reports have suggested that this may be related to the lifestyle changes such as the adoption of a western diet, higher alcohol usage, increased obesity, physical inactivity, earlier age of menarche, delayed childbearing, having fewer children and a shorter duration of breastfeeding. In contrast to incidence rates, breast cancer death rates have shown a decreasing trend in most countries, especially the high-income ones possibly due to early detection and improved breast cancer treatment. The low- and middle-income countries on the contrary are seeing an increase both in breast cancer incidence and breast cancer specific mortality.

The above trends indicate that there remains a great need to reduce the breast cancer incidence rates. Many factors, both genetic and non-genetic, determine a woman's risk of developing breast cancer and several breast cancer risk prediction models have been proposed that determine the risk. It is vitally important to risk stratify patients who have a risk of developing breast cancer as there are now effective preventive strategies available. This article summarizes the advances in the identification of women at high risk of developing breast cancer and also reviews the strategies available for breast cancer prevention.

\section{Elements of Risk and Risk Assessment}

The initial step in the assessment of breast cancer risk is a broad evaluation of the patient's personal and family history, primarily with respect to breast and/or ovarian cancers. For women not considered at risk for familial/hereditary breast cancer, an evaluation of other factors that contribute to increased breast cancer risk is recommended. Estimating the breast cancer risk for an individual patient is extremely difficult as most breast cancers are not attributable to risk factors other than female gender and increasing age. Breast cancer risk factors have historically been described as modifiable versus non-modifiable factors. The modifiable risk factors: in general are associated with lifestyle behaviors and exogenous estrogen exposure. These include physical inactivity, increased alcohol consumption, obesity, 
smoking and use of hormone replacement therapies, all of which are associated with increasing breast cancer risk. (Yang et al., 2011). The non-modifiable risk factors include increasing age, genetics, family history, increased breast density, precancerous breast lesions and prior chest wall radiation. The reproductive factors such as early onset menarche, nulliparity, first live birth after the age of 30 and late menopause are considered to be potentially modifiable risk factors. These risk factors are believed to be independently associated with a higher risk of developing breast cancer.

Breast cancer risk can also possibly be categorized as Average, High, and Very High Risk. Average Risk: A woman having no family history of breast cancer or prior history of a precancerous breast lesion is considered to be at an average risk and has a $12 \%$ lifetime risk for developing breast cancer. High Risk: The following criteria are generally used to help identify women at high risk: $(i)$ first-degree relative with a breast cancer diagnosis $<50$ years; (ii) history of atypical ductal or lobular hyperplasia (Hartman et al. 2014); (iii) 5-year Breast Cancer Risk Assessment Tool risk of $\geq 1.7 \%$ (iv) history of lobular carcinoma in situ; $(v)$ having received prior chest radiation (Travis et al., 2005); (vi) increased mammographic breast density (McTiernan et al., 2005; Boyd et al., 2007; Chiu et al., 2010; Ghosh et al., 2010; Wong et al., 2011) and (vii) International Breast Cancer Intervention Study model lifetime risk of $\geq 20 \%$. Very High Risk: Women with a strong hereditary predisposition or known $B R C A 1$ or BRCA2 mutation carriers are considered at a very high risk for developing breast cancer.

\section{Familial and Hereditary Breast Cancers}

Many women may have a family history of breast cancer but, among the majority of these women, the risk does not increase substantially and is at the most associated with doubling of the lifetime risk.

Hereditary cancers are often characterized by gene mutations associated with a high probability of cancer development, vertical transmission through either the mother or father, and an association with other tumor types. (Pharoah et al., 1997) About 90 genes/genetic loci are involved in breast cancer susceptibility. They often have an early age of onset and exhibit an autosomal dominant inheritance pattern.

Four high-penetrance genes, namely $B R C A 1, B R C A 2$, $T P 53$ and $C D H 1$ are tested in clinical practice when genetic susceptibility to breast cancer is suspected. Germ line mutations in BRCAI and TP53 are predominantly associated with infiltrating ductal carcinomas; BRCA2 mutations are associated with both ductal and lobular carcinomas, while mutations in $\mathrm{CDH} 1$ are exclusively associated with infiltrating lobular carcinomas. Mutations in PTEN and STK11 genes cause Cowden and Peutz-Jaeger syndrome respectively. (van Lier et al., 2010; Bubien et al., 2013).

Features of the family history that suggest cancer may be caused by such a high-penetrance genes include: two or more first/second-degree relatives with breast and/or ovarian cancer, breast cancer occurring before the age of
50 in a close relative, a male relative with breast cancer., ataxia-telangiectasia heterozygotes and Ashkenazi Jewish descent. In a re-analysis of fifty two case control studies comparing the cancer incidence in relatives of breast cancers, the relative risk for breast cancer increased with increasing numbers of affected first-degree relatives. Among women with one, two, and three or more affected first-degree relatives, the relative risk was 1.80,2.93, and 3.90 , respectively.

Familial cancers, on the other hand may share some but not all features of hereditary cancers. For example, although familial breast cancers occur in a given family more frequently than in the general population, they generally do not exhibit the inheritance patterns or early onset age consistent with hereditary cancers. Familial cancers may be associated with chance clustering of sporadic cancer cases within families, genetic variation in lower-penetrance genes, a shared environment, or combinations of these factors.

1. BRCA genes: The BRCA1 and BRCA2 are genes, located on chromosomes 17 and 13, respectively, with gene mutations accounting for the majority (approximately $40 \%$ ) of the inherited breast cancers. Both genes are believed to be tumor suppressor genes whose products are involved with maintaining DNA integrity and transcriptional regulation. In patients with $B R C A I$ mutations, the average cumulative risk of developing cancer by the age of 70 ranges between $55 \%$ and $85 \%$ for breast cancer and between $16 \%$ and $60 \%$ for ovarian cancer. In BRCA 2-mutation carriers, the risks ranges between $37 \%$ and $85 \%$ for breast cancer and between $11 \%$ and $27 \%$ for ovarian cancer. The mutation rates may vary by ethnic and racial groups.

Hereditary breast and ovarian cancer attributed to $B R C A 1$ or BRCA2 gene mutations can be passed on to the next generation, transmitted in an autosomal dominant pattern. The gene mutation may originate from the maternal or the paternal side, and each offspring of a BRCA carrier has a 50\% chance of inheriting the mutation. Although less than $10 \%$ of all breast cancers are linked to genetic mutations, such as BRCA-1 and BRCA-2, women who carry these mutations are at very high risk for breast cancer. The information provided by genetic testing is invaluable when making informed decisions related to breast cancer risk management. Universal genetic testing has some major drawbacks, namely the high cost and the frequency of mutations of uncertain clinical significance that occur in unselected families.

BRCA mutation testing: The BRCAPRO is a program that calculates the probability that a particular family member carries a germ-line mutation of the BRCA1 and BRCA2 genes. (Parmigiani et al., 1998) The calculations are based on Bayes' rules of determination of the probability of a mutation, given family history. The other BRCA probability tools include the Myriad I and II, Manchester, Breast and Ovarian Analysis of Disease Incidence and Carrier Estimation Algorithm (BOADICEA) (Antoniou et al., 2008) and the Ontario Family History Assessment Tool. (FHAT)

2. TP53 gene: The tumor-suppressor gene TP53 is mutated in Li-Fraumeni syndrome, which is characterized 
by early onset of a variety of tumors. The cancer susceptibility is transmitted in an autosomal dominant pattern, with a $90 \%$ lifetime risk of breast cancer. Bilateral breast cancer is noted in up to $25 \%$ of patients. They are responsible for approximately $1 \%$ of cases of familial breast cancer. Li-Fraumeni syndrome is also associated with multiple other cancers, including the SBLLA syndrome (sarcoma, breast, brain, leukemia, laryngeal and lung cancers). The cancer risk by the age of 45 years is about $41 \%$ in males and $84 \%$ in females; while the lifetime risk is $73 \%$ in males, and approaches close to $100 \%$ in females.

3. PTEN gene: PTEN mutations account for a rare genetic syndrome called Cowden disease. It is associated with intestinal hamartomas, cutaneous lesions and thyroid cancer. (Bubien et al., 2013). There is also a $30 \%$ prevalence rate of breast cancer in women with this disease. Benign mammary abnormalities (fibroadenomas, fibrocystic lesions, ductal epithelial hyperplasia, and nipple malformations) are a common association.

4. CDH1 gene: CHD1 gene is located on chromosome $16 \mathrm{q} 22$ and codes for the E-cadherin protein which is responsible for maintaining tissue integrity and mediating cell-cell adhesion. (Berx et al., 1995) The tumorsuppression role of the $C D H 1$ gene is limited to lobular breast cancer; on the contrary, its expression is unaffected in patients with ductal breast carcinomas.

5. Other Genes: Early methods such as linkage or candidate gene studies and more recent approaches like genome-wide association studies have been able to identify genomic features explaining about half of the breast cancer heritability. The Next Generation Sequencing (NGS) or second-generation sequencing technologies have the advantage that enormous numbers of sequencing reactions can be performed in parallel in a time- and cost-effective manner. This application also has incredible challenges along with promises. A number of low to moderate-effect Single Nucleotide Polymorphisms (SNPs) have also been identified as associated with breast cancer risk. Numerous studies have been conducted to investigate the association between breast cancer susceptibility with 3 polymorphism on ESR 1 gene namely PvuII (rs2234693), XbaI (rs9340799), and P325P (rs1801132) with inconsistent and controversial results. The results showed women with TT+TC or TT genotype were at a greater risk of developing breast cancer than those with CC genotype in the PvuII polymorphism. While for XbaI and P325P polymorphisms, no significance was found using any of the 3 models. (Zhang et al., 2015).

The reports from studies published showing NGS on largest number breast cancer datasets, show that although new cancer (driver) genes were identified in each of these studies, there were no new frequently mutated cancer genes shown in any of the studies. Most newly identified cancer genes were mutated in less than $10 \%$ of the patients. (Ellis et al., 2012; Shah et al., 2012; Stephens et al., 2012; Banerji et al., 2012)

Studies show that among the 100 breast cancers investigated, there were 73 different combination possibilities of mutated cancer genes, depicting a high genetic diversity. (Stephen et al., 2012). Apart from the high amount of data generated that has enabled understanding breast cancer, the interpretation of the data, with adapted informatics infrastructure, computational tools, the necessary budget, cost for NGS experimentation along with costs for data storage and analysis are to be considered if necessary for identifying a few non frequent mutations.

Therefore still a number of breast cancer susceptibility genes and loci identified with increasing use of high throughput technologies, have not been able to be used in clinical practice. This can be explained because the relative risk associated with the mutated alleles is so low that there is at best limited clinical relevance.

\section{Breast Cancer Risk Prediction Models}

Breast cancer risk assessment models estimate a woman's absolute risk of developing breast cancer either for a fixed horizon or for a woman's remaining lifetime. Breast cancer risk assessment is complicated and can be challenging as is evidenced by a number of risk assessment models. A majority of the models incorporate family history, which is the main determinant of risk, but some of these models incorporate other risk factors, such as previous abnormal breast biopsies and reproductive history.

A brief review of a few of the widely used risk assessment tools is discussed below. Health care professionals who use breast cancer risk calculation models need to be familiar with the limitations, strengths, and weaknesses of each model. It is also imperative to note that risk assessment is not a static number and patient risk changes over time; therefore, risk needs to be reassessed periodically.

1. Breast Cancer Risk Assessment Tool (Modified Gail Model): This is an interactive tool designed by the National Cancer Institute and the National Surgical Adjuvant Breast and Bowel Project to estimate a woman's risk of developing invasive breast cancer. (Gail et al., 2001). This tool was developed from the original Gail model and includes the following risk factors: current age, reproductive history, history of prior breast disease and family history, with age being the most heavily weighted risk factor. The model predicts a woman's likelihood of having a breast cancer diagnosis within the next 5 years and within her lifetime. This model does not include the age of onset of breast cancer in family members, paternal family history, or family history of ovarian cancer.

It is suitable for women $>35$ years of age with no history of ductal or lobular carcinoma in situ, no prior history of cheat wall radiation and without a strong family history of breast/ovarian cancers suggestive of a genetic predisposition. (Pankratz et al., 2008). Any woman with a 5-year risk of $\geq 1.7 \%$ determined by using this model can be considered for preventive therapy and has been used as in the major breast cancer prevention trials. The Breast Cancer Risk Assessment model was updated in 2008 to provide adjusted estimates for African American women and the Asian and Pacific Islander women.

2. Claus Model: The Claus model (Claus et al., 1994) estimates the probability that a woman developing breast 
cancer based on her family history of cancer. The can be calculated as lifetime probabilities of developing cancer or as an estimated risk that a woman will develop cancer over 10-year intervals. The Claus model may be used only for women with at least one female first- or second-degree relative with breast cancer; this model unfortunately does not take into account the other risk factors that have been associated with breast cancer, such as age of menarche, age at first live birth or a family history of ovarian cancer.

3. The International Breast Cancer Intervention Study (IBIS) model: This breast cancer risk evaluation tool also known as the Tyrer-Cuzick model, is another risk prediction algorithm for assessing breast cancer risk and the probability of harboring a BRCA mutation. (Tyrer et al., 2004). It incorporates a more extensive family history and includes reproductive risk factors and a history of benign breast disease. It is a complicated tool which is less accessible to primary care providers and is mainly utilized to determine the eligibility for enhanced screening with MRI, in addition to mammography, in women with a lifetime risk of breast cancer $\geq 20 \%$.

4. Breast Cancer Surveillance Consortium (BCSC) Risk Model: The BCSC model estimates a women's risk for developing breast cancer using breast density. Greater numbers of high-risk women eligible for primary prevention after a benign breast disease diagnosis are identified using this model. (Tice et al., 2015)

\section{Clinical Utility of Breast Cancer Risk Assessment Models}

The use of breast cancer risk assessment tools in the evaluation of risk is a good way for clinicians to engage women in a discussion of the factors that may contribute to their increased risk of developing breast cancer. Most of the clinical practice guidelines do not suggest which risk model to use, further the model predictions are limited by discordance between commonly used risk models depending on the risk factors they include and whether or not they consider the competing risk of death. (Quante et al., 2015) Women who are identified be at high risk by any of the above models should be referred to genetic counseling for a more definitive risk stratification.

\section{Genetic Counseling}

A cancer genetic counselor should be involved in determining whether a patient has a lifetime risk for breast cancer greater than $20 \%$ based on models dependent on family history. Genetic testing has major medical, psychological, ethical, legal, and social implications, in addition to the consideration of its relevance to potentially numerous family members and hence mandates referral of high-risk individuals for genetic counseling. Genetic consultation and testing are currently mainstream components of a multidisciplinary, individualized medical evaluation aimed at identification of individuals at risk for hereditary breast cancer syndromes. Genetic counselors are trained in the collection of family history and the use of models that quantify an individual's risk of a harboring a mutation. They provide pretesting education about possible outcomes of testing, the implications of both positive and negative test results for the person's health care and for her relatives and the risks and limitations of testing. Genetic counselors can additionally determine whether a person is predisposed to other hereditary gene syndromes and provide counseling on appropriate genetic testing associated with specific syndromes.

\section{Preventive Strategies}

Several research studies have assessed options aimed at reducing breast cancer risk. These have been directed at addressing the known and modifiable risk factors for breast cancer. They are generally considered suitable for high risk woman $>35$ years of age with a life expectancy of more than 10 years. In general, these strategies include: 1) Lifestyle modifications, 2) Screening strategies with Mammography/MRI 3) Chemoprevention and 4) Prophylactic (risk reducing) surgeries.

\section{1) Lifestyle Modifications}

Epidemiological studies have shown that, in general, lifestyle modification can potentially decrease the incidence of breast cancer in high-risk women. Risk modification options include regular physical activity, reduced alcohol intake, maintaining a healthy diet and body weight including avoidance of obesity and avoidance of long-term hormone replacement therapy. (Mahoney et al., 2008).

1a: Physical activity: Many studies have shown that physical activity is associated with reduced risk of developing breast cancer especially among the postmenopausal women. A study of the effect of physical activity on breast cancer risk in a population-based study of 90,509 women between 40 and 65 years of age demonstrated a $38 \%$ decrease for wom $\neg$ en who reported $>5$ hours of vigorous exercise per week when compared to women who did not participate in the recreational activities. (Tehard et al., 2006). A prospective study among 45,631 women, evaluating the association of physi $\neg$ cal activity and breast cancer risk showed that the greatest reduction in risk was in women who reported walking or hiking for 10 hours per week or more. (Howard et al., 2009). Another study showed that physical activity during the teen years delayed onset of breast cancer in BRCA mutational carriers. (King et al., 2003) Further, normal weight at menarche and low weight at age 21 years also delayed breast cancer onset in BRCA mutational carriers. (Nkondjock et al., 2006)

1b: Alcohol consumption: Numerous studies have demonstrated a consistent association between alcohol and breast cancer risk. (Mahoney et al., 2008). A metaanalysis showed a small but significant association between the risk of developing breast cancer and light alcohol intake. (Bagnardi et al., 2013). Moreover, the risk has been shown to be dose-dependent, even one drink per day modestly elevated breast cancer risk. However, the effect of a reduction in alcohol consumption on the incidence of breast cancer has not been well studied. Many 
breast cancer risk reduction panel therefore recommend that alcohol consumption should be limited to less than 1 drink per day. The panel further defined one drink as either one ounce of liquor, six ounces of wine or eight ounces of beer.

1c: Diet: Extensive research has been done to study the association between dietary factors and breast cancer risk. Some of the known associations include the increased risk with excess caloric intake, high saturated fat and excess alcohol while data on association between dietary fiber, fruits and vegetables, soy and vitamin supplements are inconclusive. (Mahoney et al., 2008; Michels et al., 2009). A few epidemiologic studies have suggested that vitamin D supplementation may have a protective role in decreasing the risk of breast cancer devel-opment. (Bertone-Johnson et al., 2005)

1d: Body Weight/ Body mass index (BMI): Many studies have established the association of high BMI and adult weight gain with increased risk for developing breast cancer in postmenopausal women. (Mahoney et al., 2008; Emaus et al., 2014). The results from the Nurses' Health Study in 87,143 postmenopausal women suggested that women experiencing a weight gain of $25 \mathrm{~kg}$ or more since 18 years of age had a greater risk for breast cancer when compared with women who have maintained their body weight. (Eliassen et al., 2006). Further, the results from a case-control study of 1073 pairs of women with BRCA mutations indicated that a weight loss of ten or more pounds among the mutational carriers between the ages 18 and 30 was associated with a decreased risk of developing breast cancer between the ages of 30 and 40 years. (Kotsopoulos et al., 2005)

1e: Hormone Replacement Therapy (HRT): HRT with estrogen and progesterone for over 5 years was found to be associated with increase in incident breast cancers and those diagnosed at a more advanced stage. (Chlebowski et al., 2010). A decision to use HRT to manage the menopausal symptoms should include a discussion of benefits and risks and should be periodically reassessed while on the medication with a plan to discontinue the medication when symptoms improve. (LaCroix et al., 2011).

1f. Breast Feeding: Breastfeeding has been shown to be protective in many studies. A review of 47 epidemiologic studies comprising of 50,302 women with invasive breast cancer and 96,973 controls estimated that for every year of breastfeeding, the relative risk of breast cancer decreases by $4.3 \%$. (Collaborative Group on Hormonal Factors in Breast Cancer 2002)

\section{2) Screening Strategies with Mammography/MRI}

Screening women with high risk of breast cancer has been an area of intense research and has evolved with the incorporation of Magnetic Resonance Imaging (MRI) screening as an adjunct to mammography. This is particularly so in women $<40$ years in whom mammography is considered less sensitive. Contrast- enhanced MRI has been shown to have a high sensitivity (86\%-100\%) for detecting breast cancer in high-risk asymptomatic and symptomatic women, although reports of specificity have been more variable (37\%-97\%)

MRI screening in addition to mammograms has been recommended for women who meet at least one of the following conditions: (a) they have a BRCA1/2 mutation, (b) they have a first-degree relative with a BRCA/2 mutation (c) their lifetime risk of developing breast cancer has been scored at 20\%-25\% (d) they have had a history of radiation to the chest wall between the ages of 10-30 or (e) they have Li-Fraumeni syndrome, Cowden syndrome, Bannayan-Riley-Ruvalcaba syndrome or one of these syndromes based on a history in a first-degree relative. (Lord et al, 2007; Saslow et al., 2007)

\section{3) Chemoprevention}

Breast cancer is a heterogeneous disease that encompasses different subtypes characterized by specific molecular biomarkers: estrogen receptor (ER)-positive, human epidermal growth factor receptor 2 (HER2)positive and triple-negative breast cancers. There seems to be a disparity in efficacy of prevention agents for each of these distinct tumor types. The chemo-prevention trials are discussed individually by each specific subtype below.

3a: Estrogen (ER) Positive Breast Cancers: The links between hormones and breast cancer has long been recognized with the identification of the estrogen receptor (ER), which has over many decades proven to be a successful target both for the treatment and prevention of breast cancer.

Selective estrogen receptor modulators (SERMs): SERMs are competitive inhibitors of the estrogen receptor and have been used effectively for decades to treat both early and advanced breast cancer. Tamoxifen acts as an estrogen antagonist in breast tissue and as an estrogen agonist in the endometrium. Conversely, raloxifene behaves as an estrogen antagonist in both the breast and the endometrium. The differences in their molecular and 3-dimensional structures affect the transcriptional activity of the activated estrogen receptor.

3a.1: First Generation Selective estrogen receptor modulator-Tamoxifen: The National Surgical Adjuvant Breast and Bowel Project (NSABP P-1) Breast Cancer Prevention Trial evaluated the use of tamoxifen for the prevention of breast cancer in high-risk women who were either pre- or postmenopausal. The study found that tamoxifen, when given for 5 years, decreased the risk for developing invasive breast cancer by $49 \%$ in women who were at an increased risk for developing breast cancer. Those with atypical hyperplasia derived the largest risk reduction: $85 \%$ (Fischer et al., 1998). In the 7-year follow-up analysis, the benefits of tamoxifen were shown to persist in women at increased risk of the disease, even after stopping therapy, with a reduction in breast cancer risk of $43 \%$. The risk remained decreased by $75 \%$ in women with atypical hyperplasia (Fischer et al., 2005). An updated analysis of the European IBIS-I trial has demonstrated that tamoxifen continues to reduce 
breast cancer risk at a median of 16 years of follow-up. (Cuzick et al., 2015).

The significant adverse effects are associated with tamoxifen, including hot flushes, vaginal dryness, increased risk of cataracts and increased incidence of the more serious but rarer side effects including deep venous thrombosis, pulmonary emboli, transient ischemic attacks, stroke and also increased risk of endometrial cancers. The serious risks are however not significantly increased in women under the age of 50 years, especially so in women without an intact uterus, thus identifying a population of women who obtain significant risk reduction benefits without incurring serious harm. The data from trials of tamoxifen in the adjuvant setting suggest a dose-dependent and duration-dependent risk of side effects; consequently, efforts are on-going to optimize the tamoxifen regimen through either dose reductions, combinations with other agents and/or topical administration. (Lazzeroni et al., 2012).

3a.2: Second generation Selective estrogen receptor modulator- Roloxifene: Treatment with the secondgeneration SERM raloxifene reportedly produced preventive effects similar to those of tamoxifen, but with reduced side effects. (Cauley et al., 2001; Vogel et al., 2006; Gardy et al., 2008; Vogel et al., 2009). In the NSABP P-2 Study of Tamoxifen and Raloxifene (STAR) trial, tamoxifen and raloxifene had equivalent effects in reducing risk of breast cancer in the high-risk postmenopausal women, which included women with a history of atypical hyperplasia or LCIS. (Vogel et al., 2006). In the updated analysis, with a median follow-up of 81 months, the benefits with tamoxifen were greater, while the risks were lower with raloxifene. (Vogel et al., 2010). Comparisons of raloxifene with tamoxifen show equal efficacy as a chemo-preventive agent for breast cancer, but there were fewer thromboembolic disorders, endometrial cancers, hysterectomies, cataracts, and cataract surgeries in women taking raloxifene, thus making raloxifene a preferred agent for postmenopausal women at high risk of breast cancer with an intact uterus. Raloxifene was however associated with hot flashes, night sweats, vaginal dryness, and weight gain.

A meta-analysis based on individual-level data from nine randomized trials (included 83,399 women with 306,617 collective years of follow-up) that compared SERMs with placebo or another drug in women without breast cancer showed a $38 \%$ reduction in the breast cancer incidence. (Cuzick et al., 2013). It further showed that 42 women needing to be treated to prevent one case of breast cancer over a ten year follow up; the largest risk reduction was in fact observed in the first five years. Also noted were a $73 \%$ increase in the incidence of thromboembolic disease with all SERMs and a $34 \%$ reduction in the incidence of non-vertebral fractures. The extended follow-up of the IBIS-I trial suggested a more favourable long term risk benefit ratio, with an estimated 22 women requiring treatment for 5 years to prevent one breast cancer in the next 20 years. (Cuzick et al., 2015). The SERMs tamoxifen and roloxifene are US FDA approved to be used as chemo-preventive agents in postmenopausal women and only tamoxifen has been studied and received an approval for breast cancer risk reduction in the hormone positive premenopausal women greater than 35 years. Unfortunately, none of the SERMbased chemo-preventive interventions decreased the risk of ER-negative breast cancers.

3a.3: Third-generation Selective estrogen receptor modulators: The third-generation SERMs i.e. lasofoxifene and arzoxifene have been investigated for their chemopreventive effects in women who are high risk for breast cancer. The Postmenopausal Evaluation and Risk Reduction with Lasofoxifene (PEARL) Trial (phase 3 trial) studied the effects of lasofoxifene, a SERM that was developed for the treatment of osteoporosis, as a chemopreventive agent in postmenopausal women with low bone mineral density. (La Croix et al., 2010). The results of this trial showed a $79 \%$ reduction in the rates of invasive breast cancer and an $83 \%$ reduction in ER-positive breast cancer among women who received lasofoxifene. A similar phase 3 trial, the Generations Trial, reported a 56\% decrease in invasive breast cancers in postmenopausal women with low bone mineral density who received arzoxifene, another SERM that was developed to maintain bone density in women with osteoporosis. (Powles et al., 2012). The third generation SERMs demonstrated an increase in the risk of venous thromboembolic, similar to tamoxifen and roloxifen. Lasofoxifene and arzoxifene are still awaiting approval for clinical use by the US FDA.

3a.4: Aromatase Inhibitors: Aromatase inhibitors block the production of estrogen in postmenopausal women and have been proven to be effective as SERMs in treating both early and advanced breast cancers. Aromatase enzyme is in fact required for the final step in estrogen biosynthesis and the third-generation aromatase inhibitors (exemestane, anastrozole, and letrozole) are potent and selective inhibitors of aromatase activity. Interest in the use of these drugs for chemoprevention developed from the findings of the Anastrozole, Tamoxifen alone and in Combination (ATAC) trial wherein postmenopausal women with earlystage breast cancer who were using anastrozole alone had a $58 \%$ reduction in contralateral invasive breast cancers. (Baum et al., 2002). A $53 \%$ reduction in breast cancer risk was also seen in the high risk women taking anastrozole in the European IBIS-II trial. (Cuzick et al., 2014). The aromatase inhibitor exemestane reduced by $65 \%$ the breast cancer risk in high-risk postmenopausal women in the Mammary Prevention 3 (MAP.3) trial. (Goss et al., 2011). The aromatase inhibitors are also being evaluated in women with a history of DCIS to determine whether they can potentially reduce breast cancer recurrences or the development of new contralateral breast tumors.

Neither exemestane nor anastrozole were associated with an increased risk of thromboembolic or cardiovascular events, or other cancers. The side effects of the aromatase inhibitors exemestane included vasomotor, sexual, and musculoskeletal symptoms. (Mounsell et al., 2014). In addition to vasomotor symptoms, musculoskeletal events (arthralgias, joint stiffness, carpal tunnel syndrome) were more common in the anastrozole arm. (Cuzick et al.2014). 
3b: Prevention of HER2 positive breast cancers: Several studies have tested the rationale of using drugs which are targeting the HER 2 oncogene as potential chemo-preventive agents in HER 2 positive breast cancers. A pilot study of the anti-HER2 drug trastuzumab was tested in patients with HER2 positive DCIS; wherein women received a single dose of trastuzumab or placebo for 14-28 days prior to their excisional surgery. Although there was no change in the size or growth rate of the excised HER2-positive DCIS; favorable immunologic responses were observed. (Kuerer et al., 2011). A few phase 2 trials are additionally testing the dual inhibitor (anti-HER2 and anti-EGFR) drug lapatinib. A phase 3 trial comparing 2 doses of trastuzumab in combination with radiation therapy versus radiation therapy alone in women with HER2-positive DCIS breast cancer is currently ongoing.

3c: Prevention of Triple negative breast cancers: Although anti-estrogen drugs have been shown to prevent ER-positive breast cancers and HER2-targeted drugs show promise in early trials for the prevention of HER2-positive breast cancers, there are no preventive interventions for the triple negative breast cancers. (ER, PR and HER2 negative) Several agents including the cyclooxygenase-2 (COX-2) inhibitor celecoxib, retinoids, statins, epigallocatechin gallate and the anti-diabetic drug are being studied as potential chemopreventive agents in preclinical and early clinical trials.

\section{Summary and Clinical Recommendations for Chemoprevention}

Many guidelines have urged clinicians to discuss the option of chemoprevention among women with increased breast cancer risk and further recommended that the discussion should include the specific risks and benefits associated with each of the chemo-preventive agents. (Vishwanathan et al., 2013). It is vital to nota that the chemo-prevention agents are recommended for women 35 years of age or older only, because its utility in women younger than 35 years is not clear. Women who have a $1.7 \%$ or greater 5 -year risk for breast cancer as determined by the modified Gail model or who have had LCIS are also potential candidates for chemoprevention [which include tamoxifen ( $20 \mathrm{mg}$ per day for 5 years), raloxifene (60 mg per day for 5 years), exemestane (25 mg per day for 5 years ) and anastrazole ( $1 \mathrm{mg} /$ day for 5 years). There is limited data are available regarding the efficacy of tamoxifen risk reduction in BRCA mutation carriers and women who have received prior thoracic radiation. The use of aromatase inhibitors to reduce breast cancer risk in premenopausal women is considered inappropriate unless it is a part of a clinical trial. It must be noted that exemestane and anastrazole are not US FDA approved for breast cancer risk reduction and further there is no robust data comparing the benefits and risks of aromatase inhibitors with either tamoxifen or raloxifene. The population of women eligible for risk reduction therapy is at a sufficiently increased risk for breast cancer to warrant a yearly bilateral mammography, clinical breast examination every 6-12 months and encouragement for breast awareness.

\section{4) Prophylactic (Risk Reducing) Surgeries}

Surgical approaches to risk reduction in high risk individuals have received a great deal of attention.

4a: Bilateral risk-reducing mastectomy: Bilateral riskreducing mastectomy has the potential to decrease the risk of developing breast cancer by at least $90 \%$ in women with very high risk including $B R C A 1$ and BRCA 2 mutation carriers as has been demonstrated in many retrospective analyses very long median follow-up periods results from smaller prospective studies have further provided support for concluding that risk reduction mastectomies protection against breast cancer in women who are mutation carriers . (Meijers-Heijboer et al., 2001; Rebbeck et al., 2001). A meta-analysis of 4 prospective studies (2635 patients) has shown a significant risk reduction of breast cancer incidence with risk reduction mastectomies among $B R C A 1$ and 2 mutation carriers (De Felice et al., 2015). It has been suggested that to prevent 1 case of breast cancer in a high risk women, the number of women needed to be treated with risk-reducing mastectomy was 6 . (Hamm et al., 1999).

Many guidelines also support the use of risk reduction mastectomies in carefully selected women at high risk for breast cancer who desire this intervention (e.g., women with a BRCA1, BRCA2, TP53, PTEN, CDH1 or STK11 mutation or possibly in women with a history of LCIS). The value of risk reduction mastectomies in women with deleterious mutations in other genes associated with a risk for breast cancer in the absence of a compelling family history of breast cancer is uncertain.

4b: Bilateral risk reducing salpingo-oophorectomy: Women with $B R C A 1$ and $B R C A 2$ mutations are at increased risk for both breast and ovarian cancers. Although the risk for ovarian cancer is lower than the risk for breast cancer among the mutation carriers, the absence of reliable methods for its early detection and the poor prognosis associated with advanced ovarian cancers have lent support for the performance of riskreducing bilateral salpingo-oophorectomy in these women after completion of childbearing. Bilateral risk-reducing salpingo-oophorectomy significantly reduces breast cancer risk by approximately $50 \%$ and ovarian cancer risk by $80 \%-95 \%$. The effectiveness of bilateral risk-reducing salpingo-oophorectomy in reducing the risk for ovarian cancer in carriers of $B R C A 1$ and $B R C A 2$ mutations has been shown in several studies, including a meta-analysis involving 10 studies of the mutation carriers. (Rebbeck et al., 2009). Although data are limited regarding an optimal age for risk-reducing salpingo-oophorectomy, the Monte Carlo simulation model provides estimates of the survival impact of breast and ovarian risk reduction strategies among the mutation carriers based on the type of BRCA mutation present, the specific risk reduction intervention and the age of the women at the time of the intervention. (Kurian et al., 2010). 
Many authors recommend limiting risk-reducing bilateral salpingo-oophorectomy to women with a known or strongly suspected BRCA 1 or BRCA 2 mutations. Peritoneal washings are recommended to be performed at the time of surgery and pathologic assessment should include serial sectioning of the ovaries and fallopian tubes. The additional benefit of concurrent hysterectomy is not clear. It is strongly recommended that women undergoing risk-reducing bilateral salpingo-oophorectomy should continue with routine health checkups and breast screening as per the standard guidelines.

Bilateral risk-reducing salpingo-oophorectomy is accompanied by menopausal symptoms, increased cardiovascular risk, impaired quality of life and accelerated bone loss. Therefore, decisions regarding the timing of bilateral prophylactic salpingo-oophorectomy and the use of hormone replacement therapy after bilateral prophylactic salpingo-oophorectomy must be made only after consultation with a multidisciplinary team. For the high risk women who are considering any of the risk reducing surgeries, it is imperative that the psychosocial issues are also addressed as such surgeries have the potential to negatively impact perceptions of one's body image, the ease of building new relationships and the quality of existing relationships. A thorough discussion with the patient of the possible alternative approaches including close surveillance, chemoprevention and participation in clinical trials is necessary to provide the patient with the full spectrum of the risk-reduction options.

\section{Barriers in Implementing Chemoprevention}

Although it is imperative that high-risk women are considered for chemoprevention, several barriers have been identified both among clinicians and high risk women that has impacted its acceptance and compliance. These include fear of possible side effects of the anti-estrogen therapies, specifically with regards to the thromboembolic events and more so relating to the risk of developing endometrial cancer. (Donolly et al., 2014). It is prudent that these unwarranted fears are overcome to enable high-risk women with a favorable risk-to-benefit ratio to be offered chemoprevention to reduce their likelihood of developing hormone receptor-positive breast cancer.

\section{Conclusions}

Breast cancer risk assessment provides a means of identifying apparently healthy women who are at increased risk for future development of this disease. Several approaches are available for identifying women with a higher than average risk of breast cancer which include an assessment of family history with genetic testing consideration, a review of clinical history, including prior breast biopsies and evaluation of mammographic density. All women need to be counseled regarding healthy lifestyle recommendations to decrease breast cancer risk. As such, management of these women require healthcare professionals to be familiar with additional risks factors so that timely recommendations can be made on surveillance/ risk-reducing strategies. The risks and benefits associated with use of each of the risk reduction agents should be evaluated and discussed with the woman as part of a shared decision-making process. Advances in breast cancer risk reduction can be better understood by encouraging the women at risk to participate in clinical trials to test new strategies for decreasing the risk for breast cancer.

\section{References}

Antoniou AC, Cunningham AP, Peto J, et al (2008). The BOADICEA model of genetic susceptibility to breast and ovarian cancers: updates and extensions. Br J Cancer, 98 , 1457-66.

Bagnardi V, Rota M, Botteri E, et al (2013). Light alcohol drinking and cancer: a meta-analysis. Ann Oncol, 24, 301-8.

Banerji S, Cibulskis K, Rangel-Escareno C, et al (2012). Sequence analysis of mutations and translocations across breast cancer subtypes. Nature, 486, 405-9.

Baum M, Budzar AU, Cuzick J, et al (2002). Anastrozole alone or in combination with tamoxifen versus tamoxifen alone for adjuvant treatment of postmenopausal women with early breast cancer: first results of the ATAC randomised trial. Lancet, 359, 2131-9.

Bertone-Johnson ER, Chen WY, Holick MF, et al (2005). Plasma 25-hydroxyvitamin D and 1,25-dihydroxyvitamin D and risk of breast cancer. Cancer Epidemiol Biomarkers Prev, 14, 1991-7.

Berx G, Staes K, van Hengel J, et al (1995). Cloning and characterization of the human invasion suppressor gene E-cadherin (CDH1). Genomics, 26, 281-9.

Boyd NF, Guo H, Martin LJ, et al (2007). Mammographic density and the risk and detection of breast cancer. $N$ Engl J Med, 356, 227-36.

Bubien V, Bonnet F, Brouste V, et al (2013). High cumulative risks of cancer in patients with PTEN hamartoma tumour syndrome. J Med Genet, 50, 255-63.

Cauley JA, Norton L, Lippman ME, et al (2001). Continued breast cancer risk reduction in postmenopausal women treated with raloxifene: 4-year results from the MORE trial. Multiple outcomes of raloxifene evaluation. Breast Cancer Res Treat, 65, 125-34.

Chiu SY, Duffy S, Yen AM, et al (2010). Effect of baseline breast density on breast cancer incidence, stage, mortality, and screening parameters: 25-year follow-up of a Swedish mammographic screening. Cancer Epidemiol Biomarkers Prev, 19, 1219-28.

Chlebowski RT, Anderson GL, Gass M, et al (2010). Estrogen plus progestin and breast cancer incidence and mortality in postmenopausal women. JAMA, 304, 1684-92.

Claus EB, Risch N, Thompson WD (1994). Autosomal dominant inheritance of early-onset breast cancer. Implications for risk prediction. Cancer, 73, 643-51.

Collaborative Group on Hormonal Factors in Breast Cancer (2002). Breast cancer and breastfeeding: collaborative reanalysis of individual data from 47 epidemiological studies in 30 countries, including 50â $€^{\wedge} 302$ women with breast cancer and $96 \hat{a} €^{\wedge} 973$ women without the disease. Lancet, 360, 187-95.

Cuzick J, Sestak I, Bonanni B, et al (2013). Selective oestrogen receptor modulators in prevention of breast cancer: an updated meta-analysis of individual participant data. Lancet, 381, 1827-34.

Cuzick J, Sestak I, Cawthorn S, et al (2015). Tamoxifen for prevention of breast cancer: extended long-term follow-up of the IBIS-I breast cancer prevention trial. Lancet Oncol, 16, 67-75. 
Cuzick J, Sestak I, Forbes JF, et al (2014). Anastrozole for prevention of breast cancer in high-risk postmenopausal women (IBIS-II): an international, double-blind, randomised placebo-controlled trial. Lancet, 383, 1041-8.

De Felice F, Marchetti C, Musella A, et al (2015). Bilateral risk-reduction mastectomy in $B R C A 1$ and $B R C A 2$ mutation carriers: a meta-analysis. Ann Surg Oncol, 22, 2876-80.

DeSantis CE, Fedewa SA, Goding Sauer A, et al (2015). Breast cancer statistics, 2015: Convergence of incidence rates between black and white women. CA Cancer J Clin.

Donnelly LS, Evans DG, Wiseman J, et al (2014). Uptake of tamoxifen in consecutive premenopausal women under surveillance in a high-risk breast cancer clinic. Br J Cancer, 110, 1681-7.

Eliassen AH, Colditz GA, Rosner B, et al (2006). Adult weight change and risk of postmenopausal breast cancer. JAMA, 296, 193-201.

Ellis MJ, Ding L, Shen D, et al (2012). Whole-genome analysis informs breast cancer response to aromatase inhibition. Nature, 486, 353-60.

Emaus MJ, van Gils CH, Bakker MF, et al (2014). Weight change in middle adulthood and breast cancer risk in the EPICPANACEA study. Int $J$ Cancer, 135, 2887-99.

Fisher B, Costantino JP, Wickerham DL, et al (2005). Tamoxifen for the prevention of breast cancer: current status of the national surgical adjuvant breast and bowel project $\mathrm{P}-1$ study. J Natl Cancer Inst, 97, 1652-62.

Fisher B, Costantino JP, Wickerham DL, et al (1998). Tamoxifen for prevention of breast cancer: report of the National Surgical Adjuvant Breast and Bowel Project P-1 Study. $J$ Natl Cancer Inst, 90, 1371-88.

Gail MH, Costantino JP (2001). Validating and improving models for projecting the absolute risk of breast cancer. $J$ Natl Cancer Inst, 93, 334-5.

Ghosh K, Vachon CM, Pankratz VS, et al (2010). Independent association of lobular involution and mammographic breast density with breast cancer risk. J Natl Cancer Inst, 102, 1716-23.

Goss PE, Ingle JN, Ales-Martinez JE, et al (2011). Exemestane for breast-cancer prevention in postmenopausal women. $N$ Engl J Med, 364, 2381-91.

Grady D, Cauley JA, Geiger MJ, et al (2008). Reduced incidence of invasive breast cancer with raloxifene among women at increased coronary risk. J Natl Cancer Inst, 100, 854-61.

Hamm RM, Lawler F, Scheid D (1999). Prophylactic mastectomy in women with a high risk of breast cancer. $N$ Engl J Med, 340, 1837-8

Hartmann LC, Radisky DC, Frost MH, et al (2014). Understanding the premalignant potential of atypical hyperplasia through its natural history: a longitudinal cohort study. Cancer Prev Res, 7, 211-7.

Hartmann LC, Sellers TA, Schaid DJ, et al (2001). Efficacy of bilateral prophylactic mastectomy in BRCA1 and BRCA2 gene mutation carriers. J Natl Cancer Inst, 93, 1633-7.

Howard RA, Leitzmann MF, Linet MS, et al (2009). Physical activity and breast cancer risk among pre-and postmenopausal women in the U.S. Radiologic Technologists cohort. Cancer Causes Control, 20, 323-33.

King MC, Marks JH, Mandell JB (2003). Breast and ovarian cancer risks due to inherited mutations in BRCAl and BRCA2. Science, 302, 643-6.

Kotsopoulos J, Olopado OI, Ghadirian P, et al (2005). Changes in body weight and the risk of breast cancer in $B R C A 1$ and BRCA2 mutation carriers. Breast Cancer Res, 7, 833-43.

Kuerer HM, Buzdar AU, Mittendorf EA, et al (2011). Biologic and immunologic effects of preoperative trastuzumab for ductal carcinoma in situ of the breast. Cancer, 117, 39-47.
Kurian AW, Sigal BM, Plevritis SK (2010). Survival analysis of cancer risk reduction strategies for $B R C A 1 / 2$ mutation carriers. J Clin Oncol, 28, 222-31.

LaCroix AZ, Chlebowski RT, Manson JE, et al (2011). Health outcomes after stopping conjugated equine estrogens among postmenopausal women with prior hysterectomy: a randomized controlled trial. JAMA, 305, 1305-14.

LaCroix AZ, Powles T, Osborne CK, et al (2010). Breast cancer incidence in the randomized PEARL trial of lasofoxifene in postmenopausal osteoporotic women. J Natl Cancer Inst, 102, 1706-15.

Lazzeroni M, Serrano D, Dunn BK, et al (2012). Oral low dose and topical tamoxifen for breast cancer prevention: modern approaches for an old drug. Breast Cancer Res, 14, 214.

Lord SJ, Lei W, Craft P, et al (2007). A systematic review of the effectiveness of magnetic resonance imaging (MRI) as an addition to mammography and ultrasound in screening young women at high risk of breast cancer. Eur J Cancer, 43, 1905-17.

Mahoney MC, Bevers T, Linos E, et al (2008). Opportunities and strategies for breast cancer prevention through risk reduction. CA Cancer J Clin, 58, 347-71.

Maresso KC, Tsai KY, Brown PH, et al (2015). Molecular cancer prevention: Current status and future directions. CA Cancer J Clin, 65, 345-83.

Maunsell E, Goss PE, Chlebowski RT, et al (2014). Quality of life in MAP.3 (Mammary Prevention 3): a randomized, placebocontrolled trial evaluating exemestane for prevention of breast cancer. J Clin Oncol, 32, 1427-36.

McTiernan A, Martin CF, Peck JD, et al (2005). Estrogen-plusprogestin use and mammographic density in postmenopausal women: women's health initiative randomized trial. J Natl Cancer Inst, 97, 1366-76.

Meijers-Heijboer H, van Geel B, van Putten WL, et al (2001). Breast cancer after prophylactic bilateral mastectomy in women with a BRCA1 or BRCA2 mutation. N Engl J Med, 345, 159-64.

Michels KB, Willett WC (2009). The Women's Health Initiative Randomized Controlled Dietary Modification Trial: a postmortem. Breast Cancer Res Treat, 114, 1-6.

Nkondjock A, Robidoux A, Paredes Y, et al (2006). Diet, lifestyle and BRCA-related breast cancer risk among FrenchCanadians. Breast Cancer Res Treat, 98, 285-94.

Pankratz VS, Hartmann LC, Degnim AC, et al (2008). Assessment of the accuracy of the Gail model in women with atypical hyperplasia. J Clin Oncol, 26, 5374-9.

Parmigiani G, Berry D, Aguilar O (1998). Determining carrier probabilities for breast cancer-susceptibility genes $B R C A I$ and BRCA2. Am J Hum Genet, 62, 145-58.

Pharoah PD, Day NE, Duffy S, et al (1997). Family history and the risk of breast cancer: a systematic review and metaanalysis. Int J Cancer, 71, 800-9.

Powles TJ, Diem SJ, Fabian CJ, et al (2012). Breast cancer incidence in postmenopausal women with osteoporosis or low bone mass using arzoxifene. Breast Cancer Res Treat, 134, 299-306.

Quante AS, Whittemore AS, Shriver T, et al (2015). Practical problems with clinical guidelines for breast cancer prevention based on remaining lifetime risk. J Natl Cancer Inst, 107.

Rebbeck TR, Friebel T, Lynch HT, et al (2004). Bilateral prophylactic mastectomy reduces breast cancer risk in BRCA1 and BRCA2 mutation carriers: the PROSE Study Group. J Clin Oncol, 22, 1055-62.

Rebbeck TR, Kauff ND, Domchek SM (2009). Meta-analysis of risk reduction estimates associated with risk-reducing salpingo-oophorectomy in $B R C A 1$ or $B R C A 2$ mutation 
Arvind Krishnamurthy et al carriers. J Natl Cancer Inst, 101, 80-7.

Saslow D, Boetes C, Burke W, et al (2007). American Cancer Society guidelines for breast screening with MRI as an adjunct to mammography. CA Cancer J Clin, 57, 75-89.

Shah SP, Roth A, Goya R, et al (2012). The clonal and mutational evolution spectrum of primary triple-negative breast cancers. Nature, 486, 395-9.

Stephens PJ, Tarpey PS, Davies H, et al (2012). The landscape of cancer genes and mutational processes in breast cancer. Nature, 486, 400-4.

Tehard B, Friedenreich CM, Oppert JM, et al (2006). Effect of physical activity on women at increased risk of breast cancer: results from the E3N cohort study. Cancer Epidemiol Biomarkers Prev, 15, 57-64.

Tice JA, Miglioretti DL, Li CS, et al (2015). Breast density and benign breast disease: risk assessment to identify women at high risk of breast cancer. J Clin Oncol, 33, 3137-43.

Travis LB, Hill D, Dores GM, et al (2005). Cumulative absolute breast cancer risk for young women treated for Hodgkin lymphoma. J Natl Cancer Inst, 97, 1428-37.

Tyrer J, Duffy SW, Cuzick J (2004). A breast cancer prediction model incorporating familial and personal risk factors. Stat Med, 23, 1111-30.

van Lier MG, Wagner A, Mathus-Vliegen EM, et al (2010). High cancer risk in Peutz-Jeghers syndrome: a systematic review and surveillance recommendations. Am J Gastroenterol, 105, 1258-65.

Visvanathan K, Hurley P, Bantug E, et al (2013). Use of pharmacologic interventions for breast cancer risk reduction: American Society of Clinical Oncology clinical practice guideline. J Clin Oncol, 31, 2942-62.

Vogel VG, Costantino JP, Wickerham DL, et al (2006). Effects of tamoxifen vs raloxifene on the risk of developing invasive breast cancer and other disease outcomes: the NSABPStudy of Tamoxifen and Raloxifene (STAR) P-2 trial. JAMA, 295, 2727-41.

Vogel VG, Costantino JP, Wickerham DL, et al (2010). Update of the National Surgical Adjuvant Breast and Bowel Project Study of Tamoxifen and Raloxifene (STAR) P-2 Trial: Preventing breast cancer. Cancer Prev Res, 3, 696-706.

Vogel VG, Qu Y, Wong M, et al (2009). Incidence of invasive breast cancer in postmenopausal women after discontinuation of long-term raloxifene administration. Clin Breast Cancer, 9, 45-50.

Wong CS, Lim GH, Gao F, et al (2011). Mammographic density and its interaction with other breast cancer risk factors in an Asian population. Br J Cancer, 104, 871-4.

Yang XR, Chang-Claude J, Goode EL, et al (2011). Associations of breast cancer risk factors with tumor subtypes: a pooled analysis from the Breast Cancer Association Consortium studies. J Natl Cancer Inst, 103, 250-63.

Zhang Y, Zhang M, Yuan X, et al (2015). Association between ESR1 PvuII, XbaI, and P325P polymorphisms and breast cancer susceptibility: a meta-analysis. Med Sci Monit, 21, 2986-96. 